\title{
Saúde sem filtro: os impactos dos filtros dos cigarros na saúde e no meio ambiente
}

\author{
Health without filters: the health and environmental impacts of \\ cigarette filters
}

André Luiz Oliveira da Silva (https://orcid.org/0000-0003-4768-959X) ${ }^{1}$

Stefania Schimaneski Piras (https://orcid.org/0000-0003-0289-0126) ${ }^{1}$

Stella Aguinaga Bialous (https://orcid.org/0000-0002-6471-5457) ${ }^{2}$

Josino Costa Moreira (https://orcid.org/0000-0002-7457-2920) ${ }^{3}$

${ }^{1}$ Agência Nacional de Vigilância Sanitária. Av. Rio Branco $14716^{\circ}$ andar, Centro. 20081971 Rio de Janeiro RJ Brasil. andre.sp.ensp@gmail.com ${ }^{2}$ University of California San Francisco, Helen Diller Family Comprehensive Cancer Center. San Francisco, CA, USA.

${ }^{3}$ Centro de Estudos da Saúde do Trabalhador e Ecologia Humana, Escola Nacional de Saúde Pública Sergio Arouca, Fundação Oswaldo Cruz. Rio de Janeiro RJ Brasil.

\begin{abstract}
Tobacco-related diseases kill eight million people worldwide ever year and are responsible for thousands of cases of cancer, cardiovascular disease and other illnesses in Brazil. Cigarette filters are believed by many to reduce the health risks of smoking. This article outlines the history of the technology of filters and discusses the impacts of these cigarette design features and their regulation. We conducted a literature review to assess the impacts of this technology. The results show that filters were initially developed for aesthetic purposes and later improved and marketed as a harm reduction technology. The most widely-used filters are those made of cellulose acetate with or without activated carbon. Despite smokers' beliefs and advertising claims, filters have no health benefits and filter tip ventilation can increase the health risks of smoking. Filters can also make cigarettes more appealing and cause significant environmental impacts. Cigarette filters have no health benefits and lull smokers into a false sense of security and should therefore be banned. Key words The tobacco industry; Control and supervision of tobacco derived products
\end{abstract}

Resumo As doenças relacionadas ao tabaco matam oito milhões de pessoas anualmente no mundo e, no Brasil, são responsáveis por milhares de casos de cânceres, doenças cardiovasculares e outras enfermidades. Os filtros nos cigarros são percebidos como uma tecnologia que reduziria os danos à saúde. O objetivo deste artigo é descrever a tecnologia dos filtros, seu histórico, seus impactos e discutir formas de regulação. Foi feita uma busca na literatura para avaliar os impactos desta tecnologia. Os resultados mostram que os filtros foram inicialmente desenvolvidos para fins estéticos, e posteriomente aprimorados e promovidos como uma tecnologia para redução de danos. O filtro de cigarro mais utilizado é o de acetato de celulose, combinado ou não com carvão ativado. Apesar das propagandas e da percepção dos fumantes, os filtros não trazem nenhum benefício à saúde, e sua associação com tecnologias como a perfuração nas ponteiras podem trazer mais riscos à saúde. Os filtros também podem tornar os cigarros mais atrativos e causam impactos ao meio ambiente. Por proporcionarem uma falsa percepção de riscos e nenhum beneficio, os filtros deveriam ser uma tecnologia proibida.

Palavras-chave Indústria do tabaco, Controle e fiscalização de produtos derivados do tabaco 


\section{Introdução}

As doenças relacionadas ao tabaco matam oito milhões de pessoas no mundo anualmente, ou seja, o consumo do tabaco e de seus derivados mata mais que Aids, cocaína, heroína, álcool, suicídios e acidentes de trânsito somados ${ }^{1}$.

No Brasil, o tabagismo é responsável por cerca de 156 mil mortes anuais. Soma-se a isso, 1.103.421 eventos médicos-ano. O tabaco ainda é responsável por cerca de 157 mil infartos agudos do miocárdio, 75 mil acidentes vasculares cerebrais e 63 mil cânceres diagnosticados anualmente ${ }^{2}$.

Assim, é natural que fumantes busquem formas de reduzir esses impactos e os filtros nos cigarros são percebidos como uma tecnologia que seria capaz de reduzir os riscos decorrentes da inalação da fumaça dos cigarros $^{3-5}$. Atualmente, alguns fabricantes sugerem que os filtros seriam capazes de reduzir as emissões de alguns componentes tóxicos da fumaça, sem, contudo, correlacioná-las com uma redução de riscos à saúde $\operatorname{dos}^{6}$. Alguns autores apontam ainda que a Indústria do Tabaco (IT) promoveu os filtros como uma tecnologia capaz de tornar os cigarros mais seguros e menos tóxicos para os fumantes, e que foi a principal tecnologia utilizada nos chamados cigarros "light".

Por outro lado, estudos independentes apontam que os filtros não são capazes de reduzir quaisquer riscos à saúde associados ao tabagismo (em algumas situações até mesmo aumentando seus riscos), seriam utilizados para estimular o consumo de cigarros, atrair novos fumantes e seriam responsáveis por um significativo impacto ambiental ${ }^{8-12}$.

O objetivo deste trabalho é descrever a tecnologia dos filtros, seu histórico, seu impacto e discutir sua regulação.

\section{Métodos}

\section{Base de dados}

Entre abril de 2019 e maio de 2019 pesquisamos a literatura científica com base principalmente no banco de dados PUBMED. Incluímos artigos relevantes citados em publicações obtidas através da pesquisa. Os sítios dos fabricantes de cigarros, assim como sites de notícias e blogs também foram pesquisados.

Fontes não revisadas por pares foram incluídas na pesquisa, pois podem fornecer dados ainda não presentes na literatura, como por exemplo a forma de como os filtros são utilizados na propaganda e as tecnologias utilizadas no mercado brasileiro.

\section{Seleção de dados, extração e síntese}

Foram buscadas publicações e documentos relacionados ao design de cigarros, marketing destes produtos, tecnologias associadas aos filtros e novos tipos de filtros.

A pesquisa foi refinada concentrando as buscas nas emissões tóxicas, na percepção e uso, impactos à saúde e ao ambiente, e na legislação relevante para estes produtos.

Realizou-se uma busca utilizando uma combinação das palavras-chave iniciais cigarro e filtro com palavras-chave relevantes para características dos filtros: cápsulas, ventilação dos filtros, aditivos, design e todas palavras-chave relevantes e pertinentes: emissões, marketing, sabor, entre outras, utilizando o método bola de neve. Não houve restrições quanto à data ou região geográfica. Somente foram selecionados resultados em inglês e português.

\section{Resultados}

Entre os artigos científicos, 93 referências foram encontradas mencionando o termo filtro de cigarro (cigarette filter), destes, 14 retornaram com o termo ventilação dos filtros de cigarros (cigarette filter ventilation), oito com o termo cápsulas nos filtros de cigarro (cigarette filter capsules), 14 com o termo aditivos nos filtros de cigarros ( $c i$ garette filter aditives) e 18 com o termo design do filtro de cigarro (cigarette filter design).

\section{Histórico}

Os filtros, nos cigarros, são utilizados desde 1860, basicamente para evitar que pedaços de tabaco entrassem na boca do fumante, para manter os lábios úmidos e destinados ao público feminino ${ }^{13-15}$. Em 1936 os filtros eram chamados de "ponteiras da beleza" (beauty tips), deixando claro para qual público se destina. O material originalmente utilizado era a cortiça, por isso muitos filtros até hoje imitam esse padrão em sua aparência ${ }^{14}$.

Nesse ínterim, foi patenteado um processo de fazer um filtro de cigarro a partir de papel crepom e, em 1935, foi desenvolvida uma máquina que possibilitava fabricar cigarros com filtro ${ }^{16}$, o que otimizou a fabricação destes tipos. 
Contudo, o uso de filtros somente se popularizou a partir da década de 1960, como resposta aos dados científicos que apontavam os danos causados pelo fumo. Diversas propagandas dos fabricantes de cigarro afirmavam que os com filtro eram mais seguros, inclusive utilizando-se da figura de médicos em suas peças publicitárias. Diversos filtros de cigarro foram desenvolvidos nessa época, aproveitando-se de um aumento da preocupação publica com os malefícios do tabaco, tendo sido utilizados inclusive filtros utilizando asbesto (amianto). Em 1980 mais de 90\% do mercado de cigarros já era constituído de cigarros com filtro ${ }^{8,13}$.

Como resposta às preocupações com a saúde, a IT priorizou estratégias bem-sucedidas de marketing focadas nos cigarros com filtro, como exemplo desse sucesso, o cigarro com filtro da marca Marlboro no início da década de 1920 era uma marca voltada para o público feminino e foi retirada do mercado durante a $2^{\text {a }}$ guerra mundial. Em meados de 1950 essa marca foi reintroduzida no mercado e reposicionada pela empresa para se tornar uma marca para o público masculino, com filtro, simbolizada pelo famoso vaqueiro das propagandas, se tornando uma das marcas mais vendidas no mundo ${ }^{17}$.

No início da década de 1970, a IT adotou a utilização da chamada perfuração nos filtros. Consiste que nas leituras realizadas nas máquinas de fumar, os cigarros cujos filtros contivessem essa tecnologia apresentavam teores menores de nicotina, alcatrão e monóxido de carbono ${ }^{8,18,19}$. Essas perfurações permitem que o ar que entra pelos orifícios dilua a fumaça da corrente principal. Contudo, em situações reais o fumante bloqueia os furos com os dedos ou os lábios na hora de fumar, fazendo com que mensurações das substâncias químicas obtidas a partir de cigarros com a perfuração de ventilação nos filtros tenham pouca relação com as condições reais de ...uso. Historicamente, em campanhas de marketing dos cigarros "lights" ou de baixos teores 8,20-23. $^{8}$.

Atualmente, os filtros apresentam uma série de cores e tecnologias como o uso de aditivos, filtros reguláveis e capsulas de sabor ${ }^{4,8,24}$.

\section{Tipos de filtros e tecnologias associadas}

Além da cortiça e do papel crepom, diversos materiais foram testados como filtros de cigarros. Espumas naturais e sintéticas, resinas, papéis especiais, algodão, seda, linho, seda de milho, outras fibras naturais, fibras sintéticas, grânulos e pós-absorventes, filtros contendo óxidos e salici- latos de alumínio, contendo tabaco cortados em pequenos pedaços foram usados, ou pelo menos sugeridos para serem usados ${ }^{8}$.

Os materiais que mais nos chamaram a atenção foram os filtros que continham asbestos, por conta da alta toxidade deste composto e que chegaram a ser comercializados ${ }^{25}$ e uma patente de um filtro para cigarro, que não se tem notícia de ter sido comercializado, feito com uma espécie de queijo ralado misturado ou não com carvão ativado $^{26}$.

Atualmente, os filtros mais utilizados são os de acetato de celulose tratados com triacetina e os de acetato de celulose combinados com carvão ativado ${ }^{6,8}$.

Os filtros de fibras de acetato de celulose têm sua eficiência na redução do material particulado influenciada por diversos fatores: tamanho, circunferência, número de filamentos de fibra e o uso de aditivos nas fibras. Os filtros combinados de acetato de celulose e carvão ativado se valem da premissa de que o carvão ativado é capaz de remover seletivamente componentes tóxicos da fumaça do tabaco ${ }^{8,27}$.

Artigos científicos e documentos revelaram que a IT havia desenvolvido cigarros para serem "elásticos" (cujos níveis de substâncias toxicas para o fumante eram maiores que aqueles aferidos pelas máquinas), com a utilização dos filtros nos cigarros para alcançar esse objetivo. Essas leituras subestimadas davam a impressão de que os produtos emitiriam menos componentes tóxicos do que os cigarros que não eram considerados "light" 28-31.

\section{Os filtros e a saúde dos fumantes}

Os fumantes percebem que os riscos associados ao tabagismo são menores em cigarros com filtros ${ }^{9,15,32}$, contudo, a literatura científica (especialmente em filtros com perfuração na ponteira) sugere o contrário ${ }^{19,33}$.

Os filtros e suas perfurações possibilitaram o surgimento dos chamados cigarros de baixos teores (lights, ultra lights, etc.) ${ }^{19}$. As propagandas apontando estes como produtos de menor risco para a saúde e a crença dos profissionais da saúde de que estes cigarros eram mais "saudáveis" que os sem filtro, tornaram esses cigarros os preferidos dos fumantes, especialmente aqueles preocupados com sua saúde ${ }^{34-37}$. Estas tecnologias também reduzem irritação da fumaça, o que contribui para a percepção menor de risco ${ }^{19,28}$.

Em relação aos impactos à saúde, a adoção de filtros e de suas perfurações geram maiores 
impactos à saúde dos fumantes pois os fumantes adaptam seus padrões de tragada para obter a inalação de maiores quantidades de fumaça de forma a garantir concentrações ótimas de nicotina, buscadas pelos fumantes ao consumir estes produtos. Essa busca por mais nicotina, levaria também à inalação (volume da tragada) de maior quantidade de outros componentes tóxicos, o que acarretaria um maior impacto à saúde dos fumantes ${ }^{28-31}$. Esse fenômeno é conhecido como efeito compensatório ou compensação ${ }^{28}$.

Quando se bloqueia as perfurações de ventilação do filtro, observa-se uma diferença de mais de dez vezes entre os valores dos teores de alcatrão, comparando-se o produto com perfuração desbloqueada contra o mesmo cigarro com a perfuração bloqueada, fazendo com que na realidade os cigarros chamados de baixos teores - ou "lights"- não sejam diferentes dos convencionais $^{18,31}$.

Estudos apontam que cigarros com filtro não trariam nenhuma redução de risco à saúde em comparação com os cigarros sem filtro ${ }^{9,38}$. A literatura sugere ainda que as perfurações de ventilação nos filtros poderiam aumentar as taxas de adenocarcinomas pulmonares, quando comparados aos cigarros sem as perfurações ${ }^{19}$. Somando-se a isso, um estudo, publicado em 2018, sugere que a remoção da ventilação dos filtros poderia reduzir os riscos de susceptibilidade ao abuso de cigarros ${ }^{39}$.

\section{Os filtros e a atratividade aos cigarros}

A ventilação dos filtros também diminuiria a irritação causada pela fumaça, tornando o produto mais palatável e atrativo, e dando a impressão ao fumante do consumo de um produto menos tóxico ${ }^{28,31}$.

Além disso, os filtros podem conter também as chamadas cápsulas de sabor, que são uma tecnologia para utilização de aditivos que possibilita o usuário a escolher se deseja o produto com sabor característico ou não. Conforme a literatura, poderiam levar um aumento da atratividade dos cigarros e reduzir a percepção de seu risco ${ }^{10,40}$.

No Brasil estas marcas com cápsulas nos filtros podem ser encontradas, com variações que incluem filtros variáveis (o fumante regularia a intensidade do sabor) e com duas cápsulas que poderiam ser esmagadas para liberar o sabor independentemente, fazendo com que ao menos em teoria o produto pudesse ter quatro sabores: sem sabor, sabor 1 ou 2 e a combinação dos dois.

\section{Impactos ambientais}

Os filtros de cigarros utilizados (ou guimbas) são os resíduos sólidos mais descartados em todo mundo, são os poluentes individuais mais encontrados nos oceanos e são praticamente onipresentes nos resíduos em todo mundo ${ }^{12}$. Estimase que 4,5 trilhões de filtros de cigarros usados sejam descartados anualmente pelo mundo ${ }^{41}$, o que representaria 845 mil toneladas de resíduos produzidos $^{42}$.

Dados do Programa Ambiental das Nações Unidas (UNEP, em inglês) ${ }^{43}$ indicam que no período de 1989-2007, os filtros de cigarros descartados foram os itens mais encontrados no International Coastal Cleanup (ICC), representando $24,6 \%$ dos itens encontrados nessas coletas ${ }^{43}$. Para se ter a ideia do impacto, o segundo item mais encontrando foram as sacolas plásticas que representaram 9,4\% dos resíduos coletados ${ }^{43}$.

A questão do descarte inapropriado dos filtros usados de cigarros levou a IT a se preocupar com essa questão e entender que, assim como os dados sobre os danos do fumo passivo levaram a legislações de ambientes livres de fumaça, a questão dos filtros descartados poderia levar a legislações ambientais mais restritivas ${ }^{44}$.

Pesquisas conduzidas nos documentos internos da IT apontaram que esta acompanhou e preparou estratégias para lidar com essa questão porque afeitaria a aceitação social do fumo, e poderia catalisar alianças entre pessoas ligadas ao controle do tabaco com ambientalistas ${ }^{44}$.

Outro ponto que merece destaque é que os filtros descartados dos cigarros não são classificados como resíduos tóxicos, entretanto os filtros estão contaminados com os diversos componentes tóxicos presentes na fumaça do tabaco, como a nicotina, nitrosaminas específicas do tabaco, fenol, formaldeído, entre outros ${ }^{45}$. Tal fato indica que estes resíduos não são devidamente tratados, mesmo quando descartados corretamente.

Alguns estudos demonstram que os filtros são tóxicos a organismos aquáticos ${ }^{41,44}$. Arsênio, nicotina, cadmio, chumbo, entre outros componentes são liberados no ambiente pelos filtros de cigarro usados.

Somando-se a estes fatores, apesar de serem foto degradáveis, os filtros usados não são biodegradáveis, o que significa que estes produtos podem ser quebrados em pedaços menores, mas efetivamente continuam no ambiente diluídos na água ou no solo ${ }^{42}$. A não biodegradação dos filtros impõe uma carga extra aos aterros sanitários, aumentam os custos da gestão de resíduos e afetam os espaços coletivos ${ }^{42}$. 


\section{Regulação dos filtros no Brasil e no mundo}

Não se tem conhecimento de nenhum local que tenha proibido a utilização de filtros nos cigarros. As tentativas (três até o momento) de proibição dos filtros identificadas foram as do estado da Califórnia (EUA), onde os danos ambientais e da saúde serviram como justificativa da medida, contudo a proposta não foi aprovada ${ }^{46}$. Foi identificado que a Alemanha proibiu o uso de cápsulas de mentol nos cigarros ${ }^{47}$.

Foi identificada também uma legislação da União Europeia sobre itens plásticos de uso único, em que os cigarros com filtros deverão apresentar rotulagem indicativa de que contém produtos plásticos de uso único e de disposição destes, além de trazer previsões de responsabilização e de ações de reciclagem dos filtros ${ }^{48}$.

Em relação à perfuração dos filtros, nenhuma proibição foi identificada, contudo, diversos au- tores consideram a perfuração de ventilação dos filtros uma tecnologia enganosa e perigosa que deveria ser proibida por enganar os consumidores, além e trazer maiores riscos à saúde $\mathrm{e}^{8,15,20-23,28,49}$.

\section{Comentários e considerações finais}

Ao considerar que os filtros dos cigarros não trazem nenhum benefício à saúde ${ }^{15,19,50,51}$, que eles têm sido utilizados como ferramenta de atração para novos fumantes, que levam à tragadas mais profundas (perfuração na ponteira do filtro), por proporcionarem uma falsa percepção de segurança e causarem impactos ao meio ambiente $\mathrm{e}^{10,12,15,28,41,52-55}$, não existe justificativa do ponto de vista da saúde e do meio ambiente para que estes componentes continuem sendo utilizados, de forma que os filtros nos cigarros deveriam ser proibidos.

\section{Colaboradores}

ALO Silva, SS Piras, SA Bialous e JC Moreira participaram da concepção, análise e redação do artigo.

\section{Agradecimentos}

Os autores agradecem à Agência Nacional de Vigilância Sanitária (Anvisa), à Fundação Oswaldo Cruz (Fiocruz), à Coordenação de Aperfeiçoamento de Pessoal de Nível Superior (Capes) e ao Conselho Nacional de Desenvolvimento Científico e Tecnológico (CNPq). Este texto representa única e exclusivamente a opinião e os pensamentos dos autores, baseados nas evidências científicas disponíveis no momento, eles não representam qualquer diretriz e/ou opinião institucional da Anvisa, da Fiocruz, do INCA, do Ministério da Saúde ou do Governo Brasileiro. 


\section{Referências}

1. World Health Organization(WHO). WHO highlights huge scale of tobacco-related lung disease deaths [Internet]. Geneva: WHO; 2019 [cited 2019 Jul 6]. Available from: https://www.who.int/news-room/ detail/29-05-2019-who-highlights-huge-scale-of-tobacco-related-lung-disease-deaths

2. Pinto MT, Pichon-Riviere A, Bardach A. Estimativa da carga do tabagismo no Brasil: mortalidade, morbidade e custos. Cad Saude Publica 2015; 31(6):1283-1297.

3. Hastrup J, Cummings K, Swedrock T, Hyland A, Pauly J. Consumers' knowledge and beliefs about the safety of cigarette filters. Tob Control 2001; 10(1):84.

4. Ford A, Moodie C, MacKintosh AM, Hastings G. Adolescent perceptions of cigarette appearance. Eur J Public Health 2014; 24(3):464-468.

5. Czoli CD, Hammond D. Cigarette packaging: Youth perceptions of "natural" cigarettes, filter references, and contraband tobacco. J Adolesc Health Off Publ Soc Adolesc Med 2014; 54(1):33-39.

6. BAT Science. Filters [Internet]. [cited 2019 Apr 2]. Available from: https://www.bat-science.com/groupms/sites/BAT_9GVJXS.nsf/vwPagesWebLive/DO7AXGBN

7. Pollay RW, Dewhirst T. The dark side of marketing seemingly "Light" cigarettes: successful images and failed fact. Tob Control 2002 Mar 1; 11(supl. 1):i18-31.

8. Hoffmann I. The changing cigarette, 1950-1995. J Toxicol Environ Health A 1997; 50(4):307-364.

9. Harris B. The intractable cigarette "filter problem." Tob Control 2011; 20 Supl. 1:i10-16.

10. Thrasher JF, Abad-Vivero EN, Moodie C, O'Connor RJ, Hammond D, Cummings KM, Yong $\mathrm{H}-\mathrm{H}$, Salloum RG, Czoli C, Reynales-Shigematsu LM. Cigarette brands with flavour capsules in the filter: trends in use and brand perceptions among smokers in the USA, Mexico and Australia, 2012-2014. Tob Control 2016; 25(3):275-283.

11. Moodie C, Thrasher JF, Cho YJ, Barnoya J, Chaloupka FJ. Flavour capsule cigarettes continue to experience strong global growth. Tob Control 2018; 28(5):595596.

12. Novotny TE, Slaughter E. tobacco product waste: an environmental approach to reduce tobacco consumption. Curr Environ Health Rep 2014;1(3):208-216.

13. Gene Borio. Tobacco History Timeline [Internet]. 2001 [cited 2019 Feb 27]. Available from: http://archive.tobacco.org/History/Tobacco_History.html

14. Kennedy P. who made that cigarette filter? The New York Times [Internet]. 2012 [cited 2018 Mar 12]. Available from: https://www.nytimes.com/2012/07/08/ magazine/who-made-that-cigarette-filter.html

15. Pauly JL, Mepani AB, Lesses JD, Cummings KM, Streck RJ. Cigarettes with defective filters marketed for 40 years: what Philip Morris never told smokers. Tob Control 2002; 11(Supl. 1):I51-61.

16. Tobacco Asia. The History of Filters [Internet]. Web Archive (recovered). 2003 [cited 2019 Apr 2]. Available from: https://web.archive.org/web/20030824115139/ http://www.tobaccoasia.com/news.asp?id=534

17. Stevenson T, Proctor RN. The SECRET and SOUL of Marlboro. Am J Public Health 2008; 98(7):1184-1194.
18. Talhout R, Richter PA, Stepanov I, Watson CV, Watson $\mathrm{CH}$. cigarette design features: effects on emission levels, user perception, and behavior. Tob Regul Sci 2018; 4(1):592-604.

19. Song M-A, Benowitz NL, Berman M, Brasky TM, Cummings KM, Hatsukami DK, Marian, C, O'Connor R, Rees VW, Woroszylo C, Shields,PG. Cigarette filter ventilation and its relationship to increasing rates of lung adenocarcinoma. J Natl Cancer Inst [Internet]. 2017; 109(12). Available from: https://www.ncbi. nlm.nih.gov/pmc/articles/PMC6059254/

20. Russell MA, Jarvis MJ, Feyerabend C, Saloojee Y. Reduction of tar, nicotine and carbon monoxide intake in low tar smokers. J Epidemiol Community Health 1986; 40(1):80-85.

21. Benowitz NL, Hall SM, Herning RI, Jacob P, Jones RT, Osman AL. Smokers of low-yield cigarettes do not consume less nicotine. N Engl J Med 1983; 309(3):13942.

22. Herning RI, Jones RT, Bachman J, Mines AH. Puff volume increases when low-nicotine cigarettes are smoked. Br Med J Clin Res Ed 1981; 283(6285):187189.

23. Kozlowski LT, Rickert WS, Pope MA, Robinson JC, Frecker RC. estimating the yield to smokers of tar, nicotine, and carbon monoxide from the lowest yield'ventilated filter-cigarettes. Br J Addict 1982; 77(2):159-165.

24. Moodie C, Angus K, Mitchell D, Critchlow N. How tobacco companies in the United Kingdom prepared for, and responded to, standardised packaging of cigarettes and rolling tobacco. Tob Control 2018; 27(e1):e85-92.

25. Longo WE, Rigler MW, Slade J. Crocidolite asbestos fibers in smoke from original Kent cigarettes. Cancer Res 1995; 55(11):2232-2235.

26. Stebbings SM. Cheese-filter cigaret [Internet]. US3234948A, 1966 [cited 2019 Apr 2]. Available from: https://patents.google.com/patent/US3234948A/en

27. Hoffmann D, Hoffmann I, Wynder EL. Lung cancer and the changing cigarette. IARC Sci Publ 1991; (105):449-459.

28. Kozlowski L, O'Connor R. Cigarette filter ventilation is a defective design because of misleading taste, bigger puffs, and blocked vents. Tob Control 2002; 11(Supl. 1):i40-50.

29. Kozlowski LT, O'Connor RJ. Official cigarette tar tests are misleading: use a two-stage, compensating test. Lancet Lond Engl 2000;355(9221):2159-2161.

30. Hurt RD, Robertson CR. Prying open the door to the tobacco industry's secrets about nicotine: the Minnesota Tobacco Trial. JAMA 1998; 280(13):1173-1181.

31. Martin Jarvis, Clive Bates. Why Low Tar Cigarettes Don't Work and How the Tobacco Industry Has Fooled the Smoking Public. [Internet]. London: Action on Smoking and Health - ASH; 1999[cited 2017 Dec 28] p. 13. Available from: http://ash.org.uk/information -and-resources/tobacco-industry-information-and -resources/low-tar-cigarettes/ 
32. O'Connor RJ, Caruso RV, Borland R, Cummings KM, Bansal-Travers M, Fix BV, King B, Hammond D, Fong GT. Relationship of Cigarette-Related Perceptions to Cigarette Design Features: Findings From the 2009 ITC U.S. Survey. Nicotine Tob Res 2013;15(11):1943-1947.

33. Stellman SD, Muscat JE, Hoffmann D, Wynder EL. Impact of filter cigarette smoking on lung cancer histology. Prev Med 1997; 26(4):451-456.

34. Centers for Disease Control and Prevention. The health consequences of smoking: a report of the surgeon general [Internet]. Washington, D.C: Dept. of Health and Human Services, Centers for Disease Control and Prevention, National Center for Chronic Disease Prevention and Health Promotion, Office on Smoking and Health; 2004 [cited 2019 Apr 2]. Report No. ISBN 0-16-051576-2. Available from: http://www.cdc. gov/tobacco/data_statistics/sgr/2004/

35. Jarvis MJ, Boreham R, Primatesta P, Feyerabend C, Bryant A. Nicotine yield from machine-smoked cigarettes and nicotine intakes in smokers: evidence from a representative population survey. J Natl Cancer Inst 2001; 93(2):134-138.

36. Institute of Medicine, Stratton K, Shetty P, Wallace $\mathrm{R}$, Bondurant $\mathrm{S}$, editors. Clearing the Smoke: assessing the science base for tobacco harm reduction. 1 edition. Washington, D.C: National Academies Press; $2001.656 \mathrm{p}$

37. World Health Organization (WHO). Scientific Advisory Committee on Tobacco Product Regulation, WHO Tobacco Free Initiative. SACTob recommendation on health claims derived from ISO/FTC method to measure cigarette yield [Internet]. Geneva: WHO; 2003 [cited 2019 Apr 2]. Available from: https://apps.who. int/iris/handle/10665/42779

38. Schulz M, Gerber A, Groneberg DA. Are filter-tipped cigarettes still less harmful than non-filter cigarettes? -a laser spectrometric particulate matter analysis from the non-smokers point of view. Int $J$ Environ Res Public Health 2016 2016; 13(4):429.

39. Stein JS, Koffarnus MN, O'Connor RJ, Hatsukami DK, Bickel WK. effects of filter ventilation on behavioral economic demand for cigarettes: a preliminary investigation. Nicotine Tob Res Off J Soc Res Nicotine Tob 2017; 20(10):1278-1282.

40. Brown J, Cohen J, Smith K. Flavor capsule cigarettes in six countries: availability by brand, variant and flavor Tob Induc Dis 2018; 16(Supl. 1):A506. [cited 2018 Oct 9]. Available from: http://www.tobaccoinduceddiseases.org/Flavor-capsule-cigarettes-in-six-countries-availability-by-brand-variant-and-flavor,83926,0,2. html

41. Slaughter E, Gersberg RM, Watanabe K, Rudolph J, Stransky C, Novotny TE. Toxicity of cigarette butts, and their chemical components, to marine and freshwater fish. Tob Control 2011; 20 (Supl. 1):i25-29.

42. Novotny TE, Lum K, Smith E, Wang V, Barnes R. cigarettes butts and the case for an environmental policy on hazardous cigarette waste. Int J Environ Res Public Health 2009; 6(5):1691-1705.

43. United Nations Environment Programme (UNEP). Marine litter: a global challenge [Internet]. Nairobi: UNEP; 2009. 232 p. [cited 2018 Oct 9]. Available from: http://wedocs.unep.org/bitstream/handle/ 20.500.11822/7787/-Marine\%20Litter_\%20A\%20 Global\%20Challenge\%20\%282009\%29-2009845 .pdf? sequence $=3$ \&isAllowed $=y$
44. Smith EA, McDaniel PA. covering their butts: responses to the cigarette litter problem. Tob Control 2011; 20(2):100-106.

45. U.S. Department of Health and Human Services. The health consequences of smoking: 50 years of progress. a report of the surgeon general [Internet]. Atlanta (GA): U.S. Department of Health and Human Services, Centers for Disease Control and Prevention, National Center for Chronic Disease Prevention and Health Promotion; 2014 Jan [cited 2016 May 16] p. 944. Available from: www.cdc.gov/tobacco

46. California Legislature. Bill Text - AB-2308 Cigarettes: single-use filters. [Internet]. Feb 13, 2018. [cited 2018 Oct 9]. Available from: https://leginfo legislature.ca.gov/faces/billTextClient.xhtml?bill_ id $=201720180$ AB2308

47. World Health Organization (WHO). Germany - Cigarettes containing menthol capsules banned [Internet]. Geneva: WHO; 2012 [cited 2018 Nov 7]. Available from: http://www.who.int/fctc/implementation/ news/news_germ/en/

48. European Union. Directive of the European Parliament and of the Council on the Reduction of the Impact of Certain Plastic Products on the Environment [Internet]. PE-CONS 11/1/19 REV 1 Jun 5, 2019. Available from: https://eur-lex.europa.eu/legal-content/EN/TX T/?uri=consil:PE_11_2019_REV_1

49. Sweeney CT, Kozlowski LT. Blocking filter vents increases carbon monoxide levels from ultralight, but not light cigarettes. Pharmacol Biochem Behav 1998; 59(3):767-773

50. O'Connor RJ, Bansal-Travers $\mathrm{M}$, Cummings KM, Hammond D, Thrasher JF, Tworek C. Filter presence and tipping paper color influence consumer perceptions of cigarettes. BMC Public Health 2015; 15:1279.

51. Strasser AA, Ashare RL, Kozlowski LT, Pickworth WB. The effect of filter vent blocking and smoking topography on carbon monoxide levels in smokers. Pharmacol Biochem Behav 2005 Oct; 82(2):320-329.

52. Novotny, TE, Bialous, SA, Burt, L, Curtis, C, Silva, VL da C e, Iqtidar, SU, Liu, Y, Pujari, S, d'Espaignet, ET. The environmental and health impacts of tobacco agriculture, cigarette manufacture and consumption. Bull World Health Organ 2015; 93(12):877-880.

53. B'chir F, Laouani A, Ksibi S, Arnaud MJ, Saguem S. Cigarette filter and the incidence of lung adenocarcinoma among Tunisian population. Lung Cancer Amst Neth 2007; 57(1):26-33.

54. Zacny JP, Stitzer ML, Yingling JE. Cigarette filter vent blocking: effects on smoking topography and carbon monoxide exposure. Pharmacol Biochem Behav 1986; 25(6):1245-1252.

55. Pauly JL, Allaart HA, Rodriguez MI, Streck RJ. Fibers released from cigarette filters: an additional health risk to the smoker? Cancer Res 1995; 55(2):253-258.

Artigo apresentado em 21/08/2019

Aprovado em 20/02/2021

Versão final apresentada em 22/02/2021

Editores-chefes: Romeu Gomes, Antônio Augusto Moura da Silva 
